Presenting Author: Marc van der Schroeff

Marc van der Schroeff

Sophia Children's Hospital, Erasmus MC

Learning Objectives: Characteristics of pediatric cholesteatoma Our experience in using a bony obliteration tympanoplasty technique is discussed.

Mastoid obliteration in cholesteatoma surgery symposium, Dutch-Belgian Otology Group.

Pediatric cholesteatoma is a different identity to its adult counterpart. It is more frequently infectious, aggressive, more proliferative and associated with less favorable prognosis. An overview on characteristics and epidemiology of pediatric cholesteatoma is given. Preliminary results on $>100$ children operated in the Sophia Children's hospital from January 2014 using predominantly a canal wall up approach with bony obliteration of the mastoid and epitympanic space are shown.

doi:10.1017/S0022215116004886

Various aspects of cholesteatoma surgery (N865)

\section{ID: 865.4}

Setup of a national multi center RCT to

evaluate the cost effectiveness of follow-up with

diffusion-weighted MRI versus 2nd look

surgery after primary cholesteatoma treatment

Presenting Author: Robert Jan Pauw

Robert Jan Pauw ${ }^{1}$, Paul Merkus ${ }^{2}$

${ }^{1}$ Erasmus MC, ${ }^{2}$ VU University Medical Center

Amsterdam

Learning Objectives: To inform on the setup of a national multi center RCT in The Netherlands that aims to evaluate the cost effectiveness of follow-up with diffusion-weighted MRI versus $2^{\text {nd }}$ look surgery after primary cholesteatoma treatment.

In The Netherlands we have set up a prospective multicenter randomized controlled trial to evaluate the cost effectiveness of follow-up with diffusion-weighted MRI versus $2^{\text {nd }}$ look surgery after primary cholesteatoma treatment. The planned start of the trial is in the $2^{\text {nd }}$ half of 2016. The aims and objectives of the trial as well as the methodology will be discussed.

\section{Research questions:}

1. Are the hearing levels after three years of follow-up with annual diffusion-weighted MRI comparable to those after follow-up with second look surgery?

2. Is a diffusion-weighted MRI follow-up strategy costeffective compared to second look surgery?

3. Are other outcome measures (residual and recurrent cholesteatoma, quality of life and adverse events) comparable between both follow-up strategies?

Hypothesis: Diffusion-weighted MRI is a cost-effective follow-up strategy after primary cholesteatoma surgery compared to the usual care, $2^{\text {nd }}$ look surgery with equal quality of care in terms of hearing, cholesteatoma detection rate, complications and quality of life.

Patients and methods: An economic evaluation alongside a prospective multicenter randomized controlled trial with an intention-to-treat analysis plus additional observational study will be performed. 132 patients of 16 years and older after primary cholesteatoma surgery treatment with normal to mild conductive hearing loss will be included and randomized in either.

'Annual diffusion-weighted MRI during 3 consecutive years, starting 1 year after primary surgery' or 'Second look surgery 1 year after primary surgery and follow-up during 3 consecutive years'.

\section{Outcome measures}

1. The degree of hearing loss after 3 thee years of follow-up.

2. The costs of three years follow-up.

3. The number of $2^{\text {nd }}$ look surgeries without cholesteatoma present (unnecessary surgical procedures) and the number of residual and recurrent cholesteatoma, health related quality of life and number of complications.

doi: $10.1017 / \mathrm{S} 0022215116004898$

\section{Free Papers (F866)}

\section{ID: 866.1}

Ouabain-Induced Cochlear Nerve

Degeneration: Synaptic Loss and Plasticity in a Mouse Model of Auditory Neuropathy

Presenting Author: Yasheng Yuan

Yasheng Yuan ${ }^{1}$, Fanglu $\mathrm{Chi}^{2}$

${ }^{1}$ EYE \& ENT Hospital of Fudan University,

${ }^{2}$ Fudan University

Learning Objectives: hair cells, ribbon synapse, neurodegeneration.

Ouabain application to the round window can selectively destroy type-I spiral ganglion cells, producing an animal model of auditory neuropathy. To assess the long-term effects of this deafferentation on synaptic organization in the organ of Corti and cochlear nucleus, and to ask whether surviving cochlear neurons show any post-injury plasticity in the adult, we quantified the peripheral and central synapses of type-I neurons at posttreatment times ranging from 1 to 3 months. Measures of normal DPOAEs and greatly reduced auditory brainstem responses (ABRs) confirmed the neuropathy phenotype. Counts of presynaptic ribbons and postsynaptic glutamate receptor patches in the inner hair cell area decreased with post-exposure time, as did counts of cochlear nerve terminals in the cochlear nucleus. Although these counts provided no evidence of new synapse formation via branching from surviving neurons, the regular appearance of ectopic neurons in the inner hair cell area suggested that neurite extension is not uncommon. Correlations between pathophysiology and histopathology showed that ABR thresholds are very insensitive to even massive neural degeneration, whereas the amplitude of ABR wave 1 is a better metric of synaptic degeneration. 